\title{
ARTICLE Heterozygous SOD2 deletion deteriorated chronic intermittent hypoxia-induced lung inflammation and vascular remodeling through mtROS-NLRP3 signaling pathway
}

\author{
Jie-qiong Song ${ }^{1,2}$, Li-yan Jiang ${ }^{1}$, Cui-ping $\mathrm{Fu}^{1}$, Xu Wu${ }^{1}$, Zi-long $\mathrm{Liu}^{1}$, Liang $\mathrm{Xie}^{1,3}$, Xiao-dan Wu ${ }^{1,3}$, Sheng-yu Hao ${ }^{1,3}$ and Shan-qun $\mathrm{Li}^{1,3}$
}

Oxidative stress caused by chronic intermittent hypoxia $(\mathrm{CIH})$ is the hallmark of obstructive sleep apnea (OSA). Among the first line of defense against oxidative stress is the dismutation of superoxide radicals, which in the mitochondria is carried out by manganese superoxide dismutase (SOD2). In this study, wild-type (WT) and SOD2-heterozygous knockout (SOD2 ${ }^{+/-}$) mice were exposed to CIH or normoxic (Nor) conditions. After 4 weeks, pulmonary artery pressure was measured, and the mice were processed to harvest either serum for cytokine assays or lungs for flow cytometry and histopathological studies. Herein, we showed that heterozygous deletion of SOD2 markedly deteriorated pulmonary remodeling and increased the oxidative stress, especially promoted the infiltration of macrophages in the lungs of $\mathrm{CIH}$ mouse. Moreover, in the intermittent hypoxia (IH)-treated RAW264.7 cells, SOD2 knockdown increased the nucleotide-binding domain-like receptor protein 3 (NLRP3) inflammasome activation accompanied with the IL-1 $\beta$ elevation and caspase-1 activity. Additionally, mitochondrial ROS (mtROS) scavenger mito-TEMPO abolished NLRP3 inflammasome activation in IH-treated RAW264.7 cells. Collectively, our results supported that SOD2 contributed to the pathogenesis of $\mathrm{ClH}$-induced lung remodeling. Meanwhile, SOD2 knockdown exacerbates oxidative damage through assembly and activation of NLRP3 inflammasome in macrophages. SOD2 may be a novel therapeutic target for $\mathrm{ClH}$-induced pulmonary inflammation and arteriole remodeling.

Keywords: SOD2; intermittent hypoxia; inflammation; NLRP3; macrophage

Acta Pharmacologica Sinica (2020) 41:1197-1207; https://doi.org/10.1038/s41401-019-0349-y

\section{INTRODUCTION}

Obstructive sleep apnea (OSA) is a common disease that affects at least $4 \%-10 \%$ of adults and has been reported to be a critical risk factor for cardiovascular morbidity [1], metabolic disorder [2], hypertension and cognitive dysfunction [3, 4]. Currently, emerging evidence has revealed that OSA is associated with lung diseases, such as chronic obstructive pulmonary disease (COPD), asthma, pulmonary arterial hypertension (PAH) and interstitial lung diseases (idiopathic pulmonary fibrosis and sarcoidosis) [5-7]. However, the precise mechanisms underlying these pathologies have not been reported.

Oxidative stress induced by nocturnal intermittent hypoxia, which results in systemic inflammation, has been identified to play a major role in OSA-related diseases [8]. Notably, the crosstalk between inflammation and OSA-related diseases is a field of active research, and chronic inflammation has been shown to aggravate target organ damage [9]. The nucleotide-binding domain-like receptor protein 3 (NLRP3) inflammasome is a cytosolic multiprotein complex consisting of the NOD-like receptor (NLR) NLRP3 scaffold, the adaptor protein apoptosis-associated speck-like protein containing a caspase recruitment domain (ASC), and pro-caspase-1. The NLRP3 inflammasome can be activated by oxidative stress and trigger the innate immune response by promoting the cleavage of caspase- 1 and the maturation of proinflammatory cytokines, such as interleukin (IL)-1 $\beta$ and IL-18 [10]. In addition, growing evidence in humans has demonstrated increased NLRP3 expression in the pathogenesis of chronic respiratory diseases and acute lung injuries [11-13]. Furthermore, previous studies have provided new insights into NLRP3 inflammasome activation in chronic intermittent hypoxia $(\mathrm{ClH})$-induced tissue damage [14]. Nevertheless, the potential role of the NLRP3 inflammasome in $\mathrm{ClH}$-induced lung damage still needs to be clarified.

Manganese superoxide dismutase (SOD2) is an important primary antioxidant enzyme located in the mitochondria and is essential for the removal of the superoxide radicals constantly being generated by the electron transport chain. The biological functions of SOD2 involve a wide spectrum of pathophysiological processes, including oxidative stress [15], inflammation [16], and metabolism [17]. Homozygous-knockout SOD2 ${ }^{-1-}$ mice are small at birth and eventually develop severe pathology in the heart and brain, leading to early death [18]. Heterozygous-knockout $\mathrm{SOD}^{+/-}$mice display a variety of clinically silent mitochondrial abnormalities underlying mitochondrial stress, resulting in an overproduction of reactive oxygen species (ROS). Furthermore,

\footnotetext{
${ }^{1}$ Department of Pulmonary Medicine, Zhongshan Hospital, Fudan University, Shanghai 200032, China; ${ }^{2}$ Department of Critical Care Medicine, Zhongshan Hospital, Fudan University, Shanghai 200032, China and ${ }^{3}$ Clinical Center for Sleep Breathing Disorder and Snoring, Zhongshan Hospital, Fudan University, Shanghai 200032, China Correspondence: Sheng-yu Hao (janet9yu@163.com) or Shan-qun Li (li.shanqun@zs-hospital.sh.cn)

These authors contributed equally: Jie-qiong Song, Li-yan Jiang, Cui-ping Fu
}

Received: 26 August 2019 Accepted: 12 December 2019

Published online: 17 February 2020 
1198

abnormalities in SOD2 function are involved in the pathogenesis of lung diseases, such as $\mathrm{PAH}$, lung cancer, and radiation-induced pulmonary injury $[16,19,20]$. In fasting mice, knocking down SOD2 expression increases NLRP3 formation and activation, and reduced SOD2 activity is accompanied by increased ROS levels and hyperactivity of NLRP3 in macrophages [21]. Furthermore, hypoxia primes and activates NLRP3 inflammasome activity in many different cell lines and human macrophages [22, 23]. Nevertheless, the relationship between SOD2 and NLRP3 in $\mathrm{CIH}-$ induced lung damage has not been explored. Based on the fact that oxidative stress and inflammatory cell infiltration participate in $\mathrm{ClH}$-associated damage, we hypothesize that SOD2 plays an important role in $\mathrm{ClH}$-induced lung injury.

In this study, using cultured cells and a $\mathrm{ClH}$ mouse model, we evaluated the effect of SOD2 knockdown on oxidative stress activities and inflammatory injury in targeted lung tissues exposed to $\mathrm{ClH}$. We also explored the SOD2/NLRP3 mechanism and cellular immune mediators involved in $\mathrm{ClH}$-induced lung damage. An improved mechanistic understanding of $\mathrm{ClH}$-induced pulmonary inflammation and remodeling is a prerequisite for the development of more effective therapies for OSA-related lung diseases.

\section{MATERIALS AND METHODS}

Animals and $\mathrm{ClH}$ exposure

WT control and SOD2 ${ }^{+/-}$mice (both on the C57BL/6 background) were purchased from the Model Animal Research Center of Nanjing University and were bred and housed in the veterinary facility of the Experimental Animal Center of Fudan University. This study was carried out according to a protocol approved by the Ethics Committee of the Zhongshan Hospital, Fudan University. In vivo $\mathrm{CIH}$ research was performed as previously described [24]. Briefly, 8-week-old male mice were exposed to a 1-min-period IH cycle in a designed chamber, and the oxygen concentration was adjusted between $4 \%$ and $21 \%(\mathrm{ClH}$ group; $n=6)$, or the same chamber was open to room air (Nor group; $n=6$ ). Nitrogen was delivered to the chamber at a rate sufficient to achieve a fraction of inspired oxygen $\left(\mathrm{FiO}_{2}\right)$ of $4 \%-7 \%$ within $30 \mathrm{~s}$ and maintain this $\mathrm{FiO}_{2}$ level for $10 \mathrm{~s}$; then, oxygen was introduced to achieve an $\mathrm{FiO}_{2}$ of $20 \%-21 \%$ within $30 \mathrm{~s}$. This $\mathrm{IH}$, which mimicked a rate of 60 apneas/h, which is typical of severe OSA, was applied for $8 \mathrm{~h} /$ day (8:00-16:00) for 4 weeks. All in vivo experiments were repeated at least three times.

\section{Human samples}

Patients with OSA were recruited from outpatients at the Clinical Centre for Sleep Breathing Disorder and Snoring of Zhongshan Hospital between February 2018 and September 2019. All patients were given diagnoses based on the results of polysomnography. The apnea-hypopnea index (AHI) was defined as the number of episodes of obstructive apnea and obstructive hypopnea per hour of sleep. An $\mathrm{AHI} \geq 5$ was taken as the threshold for OSA, and the severity of OSA was graded by the AHI (mild: 5-15 events per hour, moderate: between 15 and 30 events per hour, and severe: $\geq 30$ events per hour). Age-matched controls had AHls of less than five events per hour. Serum was obtained from the OSA patients $(n=29)$ and controls $(n=10)$. The study was approved by the ethics committee of Zhongshan Hospital affiliated with Fudan University (approval number B2018-216R).

\section{Pulmonary arterial pressure measurement}

At the end of the 4-week $\mathrm{ClH}$ exposure period, a longitudinal skin incision was made along the right side of the neck, and blunt layer-by-layer separation of the tissues was performed until the right external jugular vein was exposed. A Mikro-Tip ${ }^{\circledR}$ catheter with a transducer (Millar, Inc., 6001-A Gulf Freeway Houston, USA) was gradually inserted into the pulmonary artery through an incision in the right external jugular vein of the mouse, and the right ventricular systolic pressure was recorded. At the end of the experiment, the mice were euthanized with an intraperitoneal injection of $3 \%$ sodium pentobarbital, and serum/plasma and tissue samples were harvested.

\section{Immunohistochemistry}

After flushing, the upper lobe of each lung was stored at $-80^{\circ} \mathrm{C}$ for RNA and proteomic analyses. The lower lobe of the left lung was fixed by perfusion with $3.8 \%$ paraformaldehyde and embedded in paraffin. Tissue sections $(4 \mu \mathrm{m})$ were stained with hematoxylin and eosin (HE) or Masson's trichrome for routine histological analysis. For histopathological analysis, the total tissue on slides was scored by two blinded pathologists with expertise in lung pathology. The degree of microscopic injury was evaluated on the basis of the following variables: alveolar and interstitial thickness, inflammatory infiltration, and collagen deposition referring to a reference [25]. A total of three slides from each lung sample were randomly screened, and the mean was obtained as the representative value of the sample.

Cell culture and $\mathrm{ClH}$ modeling in vitro

RAW264.7 cells were purchased from the Chinese Academy of Medical Science (Shanghai, China) and cultured in Dulbecco's Modified Eagle's Medium (DMEM) supplemented with $10 \%$ fetal bovine serum (FBS), $100 \mathrm{U} / \mathrm{mL}$ penicillin, $100 \mathrm{U} / \mathrm{mL}$ streptomycin, and $3 \mathrm{mM}$ glutamine. Cells were cultured in a designed computercontrolled incubator chamber (Biospherix, Lacona, NY), where the $\mathrm{O}_{2}$ concentration varied between 0 and $21 \%$ every 30 min by injecting $\mathrm{N}_{2}$ or $\mathrm{O}_{2}$ with $5 \% \mathrm{CO}_{2}$. The dissolved $\mathrm{O}_{2}$ inside the culture medium was monitored by a laser $\mathrm{O}_{2}$ probe (Biospherix), and the $\mathrm{IH}$ reached $5 \% \mathrm{O}_{2}$ and $21 \% \mathrm{O}_{2}$ as the hypoxic and normoxic values according to the sensing of the cells. Normal air conditions corresponded to $21 \%$ $\mathrm{O}_{2}$ and $5 \% \mathrm{CO}_{2}$. All in vitro experiments were repeated at least three times.

\section{LV-shSOD2 transfection and quantitative real-time PCR}

Cells were plated in 24 -well $\left(1.0 \times 10^{4}\right)$ or six-well plates $\left(1.0 \times 10^{6}\right)$ and cultured overnight before transfection. The cells were transfected with LV-shSOD2 (target sequence, 5'-GCCACATTAACGCGCAGAT-3') or LV-NC according to the manufacturer's protocols (Biolink, Shanghai, China). Briefly, cells grown to $30 \%$ $-40 \%$ confluence were transduced with LV-shSOD2 or LV-shNC $(\mathrm{MOI}=100)$ in a total volume of $300 \mu \mathrm{L}$ (24-well plate) or $1 \mathrm{~mL}$ (sixwell plate) of DMEM (10\% FBS). After $12 \mathrm{~h}$, the cells were washed extensively with phosphate buffer saline (PBS) to remove lentiviral genomic RNA, and fresh medium was added. The cells were maintained in culture for another $48 \mathrm{~h}$. The knockdown efficiency of LV-shSOD2 was detected by Western blot analysis. After $48 \mathrm{~h}$ of transfection, the cells were exposed to $\mathrm{IH}$ or normoxia for different durations and harvested for the following experiment. Total RNA was extracted from lung tissue samples and cultured RAW264.7 cells using TRIzol reagent (Takara Bio, Shiga, Japan). cDNA was synthesized from the isolated RNA, and quantitative PCR was performed according to the manufacturer's instructions. This quantitative assay was performed using an SYBR QPCR kit (Toyobo, Osaka, Japan). The primer sequences used for PCR were as follows: SOD1, sense: $5^{\prime}$-CTCAGGAGAGCATTCCATCATT-3' and anti-sense: 5'-CTCCCAGCATTCCAGTCTT-3'; SOD2, sense: 5'-CA CATTAACGCGCAGATCATG-3' and anti-sense: ${ }^{\prime}$-CCAGAGCC TCGTGGTACTTCTC-3'; SOD3, sense: 5'- CTTGTTCTACGGCTTGCT ACT- $3^{\prime}$ and anti-sense: $5^{\prime}$-CTTGTTCTACGGCTTGCTACT-3'; IL-1 $\beta$, sense: 5'- TTCAGGCAGGCAGTATCACTC-3' and anti-sense: 5'-GA AGGTCCACGGGAAAGACAC-3'; and IL-18, sense: 5'-CCACCC TACGAAGTACCAACT-3' and anti-sense: 5'-GCCAGAATGATGTGA TACTGGG-3'.

Determination of oxidative stress factor and cytokine production Lung tissue samples from mice were homogenized in a lysis buffer and centrifuged at $10,000 \times g$ for $10 \mathrm{~min}$ at $4{ }^{\circ} \mathrm{C}$. The activities of 
SOD2 or SOD were detected using commercial kits (Beyotime, China) following the manufacturer's instructions. All results were normalized to the protein concentration and are expressed as $\mathrm{U} / \mathrm{mg}$ of protein as appropriate. For mouse lung sections, DHE was used at a final concentration of $50 \mu \mathrm{M}$ on tissue embedded in OCT. The sections were incubated for $7 \mathrm{~min}$ at $37^{\circ} \mathrm{C}$ in the dark and washed twice with PBS. The slides were covered and immediately mounted with Fluoroshield mounting medium with DAPI (no. ab104139). To detect cellular mitochondrial ROS (mtROS), cells were treated with mitoSOX ${ }^{\mathrm{TM}}$ red mitochondrial superoxide indicator (Invitrogen ${ }^{\mathrm{TM}}$ ) at a final concentration of $5 \mu \mathrm{M}$ for $10 \mathrm{~min}$ at $37^{\circ} \mathrm{C}$ and washed with PBS three times. The cells were detected by the quantitation of fluorescence intensity by flow cytometry. All data were analyzed with FlowJo software (Tree Star Inc., San Carlos, CA).

Measurement of mitochondrial membrane potential

Mitochondrial membrane potential was measured using the potential-sensitive dye JC- $1 \quad\left(5,5^{\prime}, 6,6^{\prime}\right.$-tetrachloro- $1,1^{\prime}, 3,3^{\prime}$-tetraethyl-benzimidazolylcarbocyanine iodide). Briefly, RAW264.7 cells $\left(1 \times 10^{4}\right)$ were plated in a 24-well plate and allowed to attach overnight. shNC or shSOD2 lentiviruses were added into the wells and cultured for $48 \mathrm{~h}$ under normoxia, and the cells were then exposed to IH for $48 \mathrm{~h}$ with or without mito-TEMPO (Santa Cruz, sc-221945) treatment ( $100 \mathrm{nM}, 2 \mathrm{~h}$ before $\mathrm{IH}$ exposure). The cells were incubated with medium containing $\mathrm{JC}-1 \quad(10 \mu \mathrm{g} / \mathrm{mL})$ for $20 \mathrm{~min}$ at $37^{\circ} \mathrm{C}$. Then, the cells were washed with PBS and observed by confocal microscopy or measured by the quantitation of fluorescence intensity through flow cytometry.

\section{Flow cytometry}

At sacrifice, the right lung was dissected into single lobes and then digested with an enzyme mixture (buffer $S$, enzyme $D$, and enzyme A from Miltenyi Biotec, Bergisch Gladbach, Germany). After filtration and centrifugation, the resulting cell suspensions were filtered through a 70- $\mu \mathrm{m}$ nylon mesh, and red blood cells were lysed with a cell lysing solution (BD Biosciences Pharmingen, San Diego, CA, USA). Then, the cells were incubated in saturating doses of purified anti-mouse Fc receptor (anti-CD16/32, clone 2.4G2, BD Bioscience) in $1 \mathrm{~mL}$ of PBS $+5 \%$ FCS for 10 min on ice to prevent antibody binding to the Fc receptor. The following antibodies were used for surface staining (eBioscience): PE-labeled anti-CD45, FITC-labeled anti-CD11b, Horizon ${ }^{\text {TM }}$ BV480-labeled antiCD11C, PerCP-Cy5.5-labeled anti-Ly6C and PE-Cy7-labeled antiLy6G. A PE annexin V apoptosis detection kit was used to detect apoptosis in RAW264.7 cells according to the manufacturer's protocol.

\section{ELISA}

Commercially obtained ELISA kits were used to quantify the levels of immunoreactive mouse IL-1 $\beta$ (RayBiotech, Inc., USA) released from cells into the culture medium under various experimental conditions. The culture medium was collected, centrifuged at $14,000 \mathrm{rpm}$ for $5 \mathrm{~min}$ at $4{ }^{\circ} \mathrm{C}$, and processed according to the manufacturer's instructions in at least three independent experiments.

\section{Western blot analysis}

Western blot analysis of cell and tissue lysates was performed as previously described [13]. Briefly, membranes were blocked with $5 \%$ nonfat dry milk in TBST for $1 \mathrm{~h}$ and incubated overnight at $4{ }^{\circ} \mathrm{C}$ with primary Abs against SOD2 (Cell Signaling, Danvers, MA), NLRP3, ASC, pro-caspase-1, cleaved caspase-1, ACTB and GAPDH (Santa Cruz Biotechnology, Santa Cruz, CA, USA). The membranes were incubated with an anti-rabbit horseradish peroxidaseconjugated secondary Ab (Cell Signaling), and protein bands were detected by chemiluminescence using Immobilon Forte Western HRP substrate (Millipore WBLUF0500). Densitometry was performed using ImageJ software (NIH, Bethesda, MD). All experiments were performed in triplicate.

Statistical analyses

All statistical analyses were performed using SPSS version 18.0 (SPSS Inc., IL, Chicago, USA). The data are expressed as the mean \pm SEM. Student's $t$ test was used for comparisons between two groups, whereas analysis of variance (ANOVA) with Dunnett's test was used for comparisons of more than two groups. Correlations between SOD and the $\mathrm{AHI}$ in serum samples from patients were assessed with the Pearson correlation coefficient. Differences were considered significant at $P<0.05$. All results represent at least three independent replicates.

\section{RESULTS}

SOD2 expression is decreased in OSA patients and $\mathrm{CIH}$ mice First, we investigated SOD2 activity in serum samples from controls $(n=10)$ and OSA patients $(n=29)$. We found a negative correlation between SOD2 activity and the AHI $(P<0.05$, $r=-0.462$, Fig. 1a). To investigate the effect of SOD2 deficiency on model mouse lungs, we used $\mathrm{SOD} 2^{+/-}$mice and a widely accepted OSA mouse model called the $\mathrm{CIH}$ model, in which mice were exposed to $\mathrm{CIH}$ conditions for 4 weeks as described in the Methods section. SOD2 ${ }^{+/-}$and WT mice were genotyped to confirm the presence of one or two copies of the SOD2 gene, respectively (Fig. 1b). We then evaluated the effect of $\mathrm{CIH}$ on the expression levels of mammalian superoxide dismutases, including SOD1, SOD2, and SOD3, in the WT and SOD2 ${ }^{+/-}$mice. The mRNA level of SOD1 in the lungs was not significantly different between the WT and SOD2 ${ }^{+/-}$mice, and $\mathrm{ClH}$ exposure had no effects on SOD1 expression in the WT and SOD2 ${ }^{+1-}$ mice (Fig. 1c). SOD3 mRNA expression was not different between the WT and SOD2 ${ }^{+/-}$ mice, while $\mathrm{CIH}$ exposure increased the SOD3 mRNA level in the WT and SOD2 $2^{+/-}$mice (vs. the WT group, $P<0.01$ ). Although the mRNA level of SOD3 was elevated in the WT and SOD2 $2^{+1-}$ mice under $\mathrm{CIH}$ conditions, total SOD activation was decreased in the $\mathrm{CIH} \mathrm{SOD} 2^{+/-}$mice compared with the CIH WT mice $(P<0.05$, Fig. 1d). Notably, the basal levels of SOD2 mRNA (Fig. 1c) and protein (Fig. 1e, f) were decreased in the SOD2 ${ }^{+/-}$(vs. WT, $P<$ 0.05 ) mice, and $\mathrm{CIH}$ exposure further lowered SOD2 expression in the SOD $2^{+/-}$mice (vs. CIH WT, $P<0.01$ ).

\section{Heterozygous SOD2 deletion exacerbates pulmonary vascular remodeling in $\mathrm{CIH}$ mice}

Since our previous study demonstrated that $\mathrm{CIH}$ exposure induced $\mathrm{PAH}$ in rats, we next evaluated $\mathrm{PAH}$ and lung vascular remodeling in WT and SOD2 ${ }^{+/-}$mice under Nor or $\mathrm{CIH}$ conditions. There were no discernible differences in PAH between the WT and SOD2 ${ }^{+/-}$ mice under normoxia. However, the WT mice showed increased RV pressure (RVP) values from $20.5 \pm 0.645$ to $46.67 \pm 1.944 \mathrm{mmHg}$ after being exposed to $\mathrm{ClH}$ for 4 weeks $(P<0.001)$. In contrast, the $\mathrm{CIH}$ SOD2 ${ }^{+/-}$group showed significantly elevated RVP values from $46.67 \pm 1.944$ to $78.15 \pm 1.737 \mathrm{mmHg}$ (vs. ClH WT, $P<0.001$, Fig. 2a). The remodeling of pulmonary arterioles is the main characteristic of $\mathrm{PAH}$. Therefore, morphometric analyses of lung tissue samples were performed by HE staining to detect the impact of heterozygous SOD2 deletion on the pulmonary arterioles under the $\mathrm{CIH}$ conditions (Fig. 2b). Compared with the Nor group, the $\mathrm{ClH}$ group showed an increased degree of vascular muscularization as indicated by the thickness of the vascular wall $(P<0.01$, Fig. $2 \mathrm{~b}, \mathrm{c})$, whereas the $\mathrm{ClH} \mathrm{SOD2}{ }^{+/-}$group presented with more severe vascular thickness than the $\mathrm{CIH}$ WT group $(P<0.05)$. The hyperplasic state of collagen was observed by Masson's trichrome staining. The collagen volume fraction was greater in the $\mathrm{CIH}$ group than in the Nor group, and the change was exacerbated in the $\mathrm{CIH} \mathrm{SOD} 2^{+/-}$mice (Fig. $2 \mathrm{~b}, \mathrm{~d}$ ). These results indicated that pulmonary vascular remodeling was 
a

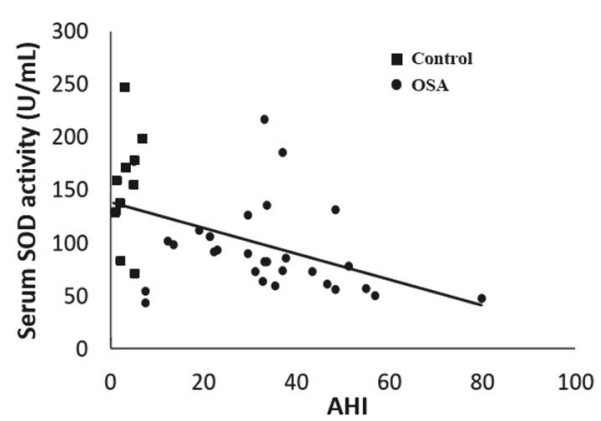

b

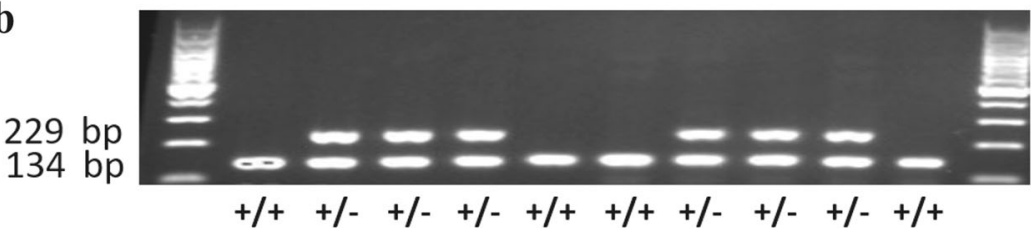

c

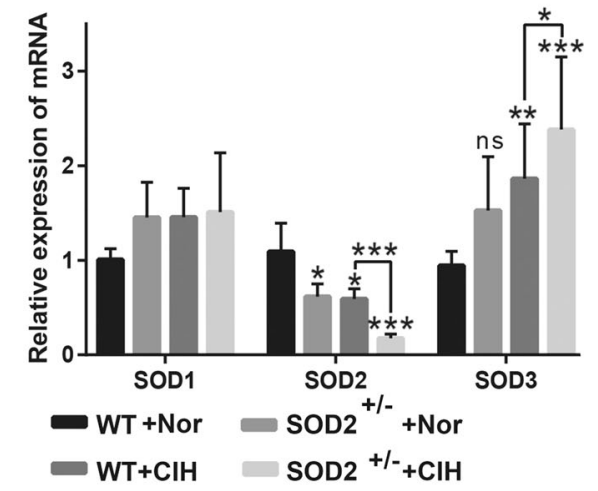

e

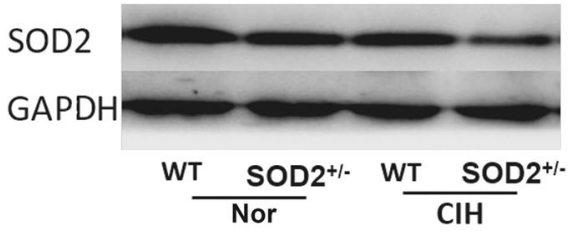

d 100

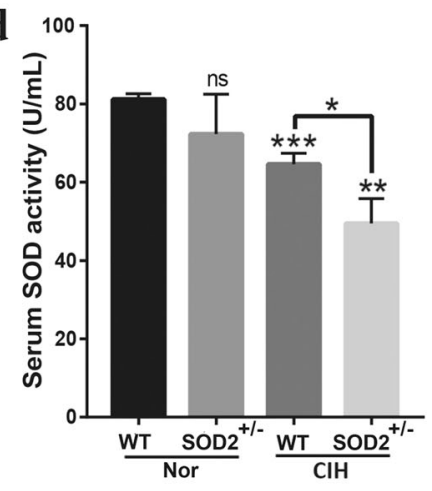

f

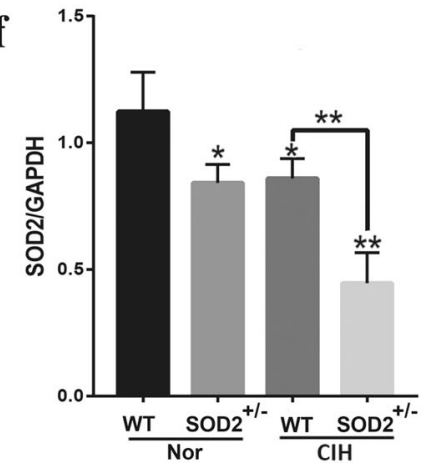

Fig. 1 Expression and activity of superoxide dismutase in OSA patients and CIH-treated mice. a ELISA analysis was performed to investigate the correlation between the AHI and SOD2 activity in serum samples from ten control individuals and 29 OSA patients. b Total DNA was used as a template for genotyping SOD2 ${ }^{+/-}$and WT mice. The amplification of a 229-bp band indicated the integration with the SOD2 gene. c Reverse transcription quantitative PCR (RT-qPCR) evaluation of the pulmonary mRNA levels of SOD1, SOD2 and SOD3 was performed with the lungs of WT and SOD2 ${ }^{+/-}$mice exposed to Nor or $\mathrm{CIH}$. d SOD levels in serum samples collected from the four mouse groups were detected by ELISA. e Representative Western blot images for the pulmonary levels of SOD2 and GAPDH are shown. f Densitometric quantification of relative SOD2 expression is shown. We analyzed more than six individual mouse serum or tissue samples in every experiment. Independent experiments were repeated three times, and a representative figure is shown. The data are shown as the mean \pm SEM of one experiment with six mice. ${ }^{*} P<0.05,{ }^{* *} P<0.01$, and ${ }^{* * *} P<0.001$ compared with the WT group. WT wild type, AHI apnea hypoxia index, Nor normoxia, $\mathrm{ClH}$ chronic intermittent hypoxia.

more severe in $\mathrm{SOD}^{+/-}$mice than in WT mice under the $\mathrm{ClH}$ conditions.

Heterozygous SOD2 deletion exacerbates lung inflammation and oxidative stress in $\mathrm{ClH}$ mice

It is widely accepted that oxidative stress contributes to lung inflammation, and SOD2 is an essential antioxidant that detoxifies free superoxide radicals. Therefore, we determined whether SOD2 deficiency exacerbates inflammation and ROS production in lung tissue samples from the $\mathrm{CIH}$ model. $\mathrm{CIH}$ induced obvious lung injury, evidenced by a thickened alveolar wall, inflammatory cell infiltration, and collagen deposition, while the Nor group exhibited normal histomorphology (Fig. 3a). ClH-induced lung injury was further deteriorated by heterozygous SOD2 deletion. These changes were verified by statistical histological scoring and are presented in Fig. 3b. In addition, we found that the lung mRNA 
a

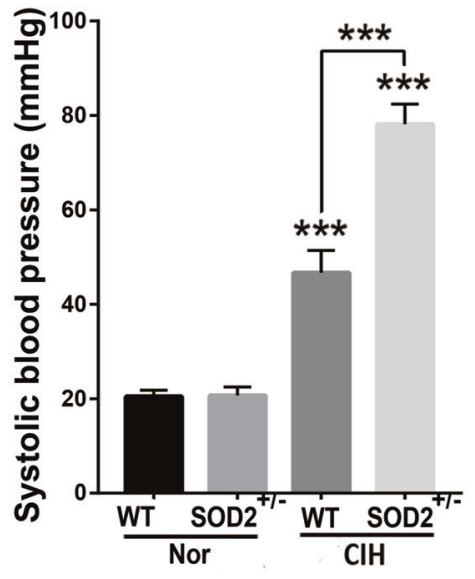

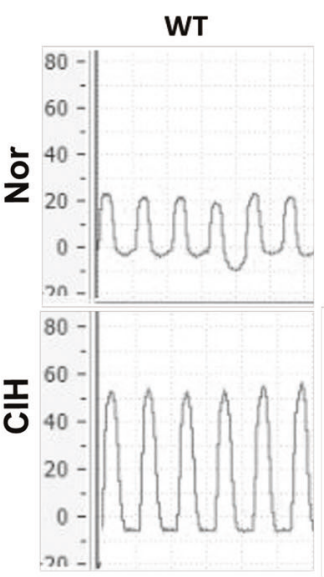

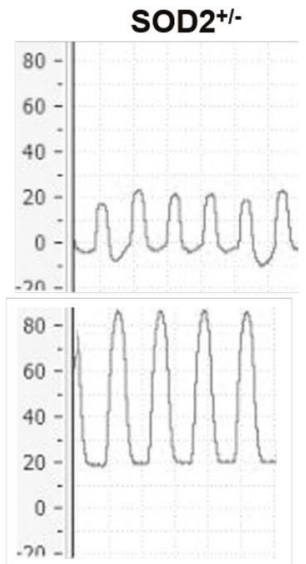

b

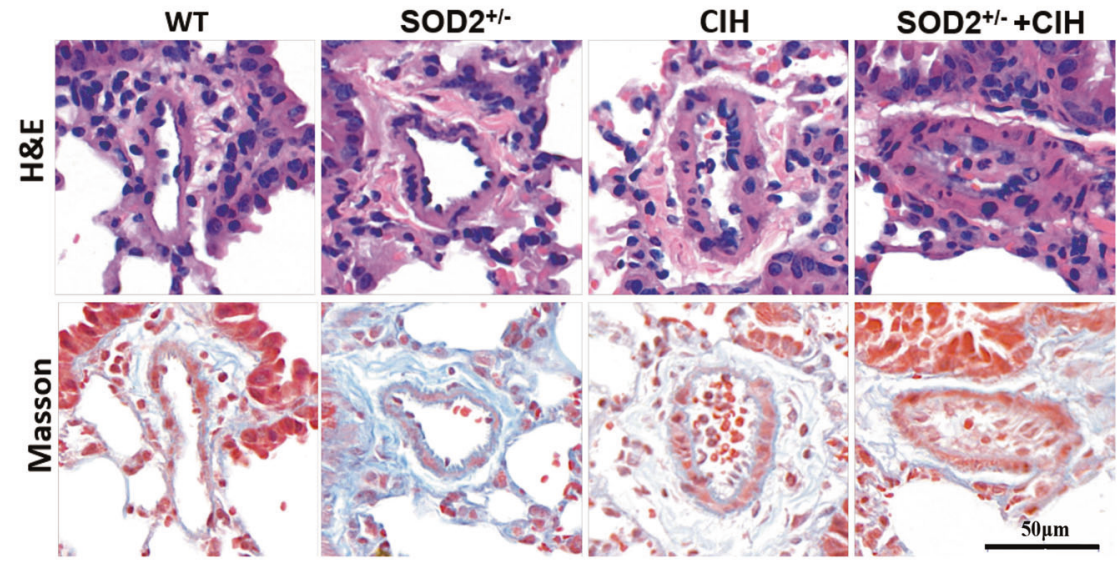

c

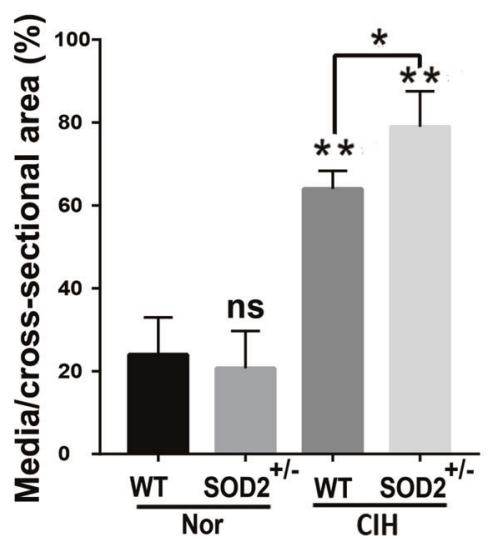

d

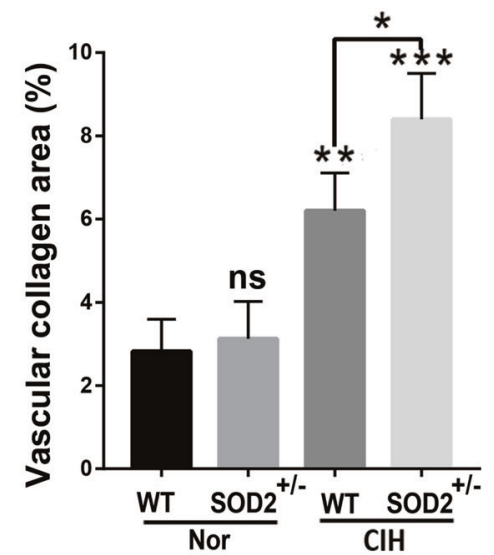

Fig. 2 Deficiency in SOD2 deteriorates CIH-induced pulmonary artery remodeling. WT and SOD2 ${ }^{+/-}$mice were exposed to Nor or CIH conditions for $\mathbf{4}$ weeks. a Systolic blood pressure was then measured, and representative photomicrographs are presented. b HE and Masson's trichrome staining of pulmonary arterioles in the four groups were performed and are presented. c The percentage wall thickness of pulmonary arterioles was defined as the area occupied by the vessel wall divided by the total cross-sectional area of the arteriole. d The vascular collagen area was evaluated by quantitative image analysis of Masson's trichrome staining. Scale bar: $50 \mu \mathrm{m}$. Graph bars represent the mean \pm SEM of 20 vessels from 6 mice per group. ${ }^{*} P<0.05$, ${ }^{*} P<0.01$, and ${ }^{*} * P<0.001$ compared with the WT group. Nor normoxic, CIH chronic intermittent hypoxia, HE hematoxylin and eosin, WT wild type.

levels of proinflammatory cytokines, such as $I L-1 \beta$ and $I L-18$, were significantly increased in the CIH WT group (vs. WT, $P<0.05$ ), while SOD2 deficiency further exacerbated lung proinflammatory cytokine levels under the $\mathrm{ClH}$ conditions (vs. ClH WT, $P<0.05$, Fig. 3c). Since OSA often causes target-organ injury through increased oxidative stress, we further determined the relative levels of lung oxidative stress in each experimental group. Lung ROS stained by DHE demonstrated that $\mathrm{ClH}$ exposure caused a significant increase in ROS production in both WT and SOD2 ${ }^{+/-}$ mice, but the increase was greater in the SOD2 ${ }^{+/-}$mice (Fig. $3 \mathrm{~d}$, $P<0.05)$.

Heterozygous SOD2 deletion modulates macrophage subsets in response to $\mathrm{ClH}$ exposure

Macrophages are considered the primary source of proinflammatory cytokines and play an important role in the response to hypoxic stress during pulmonary pathogenesis [26]. Macrophages were gated based on the differential expression of CD11b and 
a

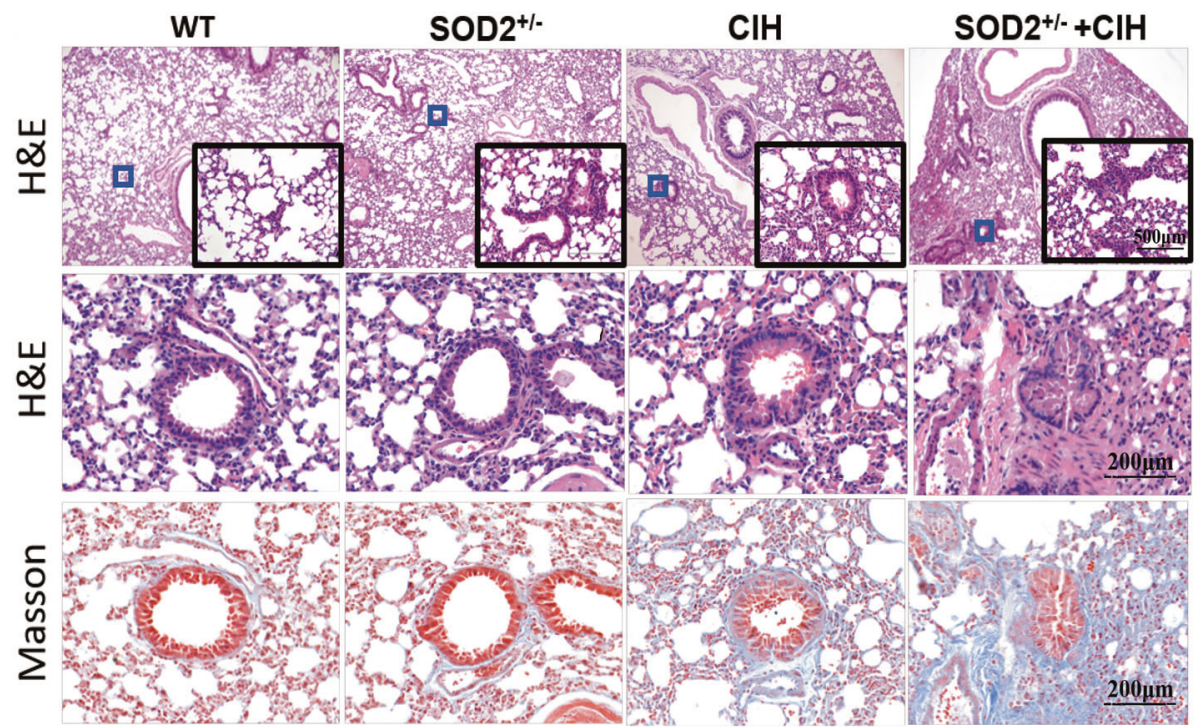

b

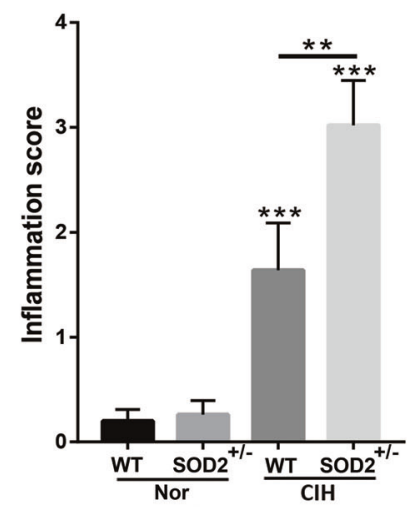

c

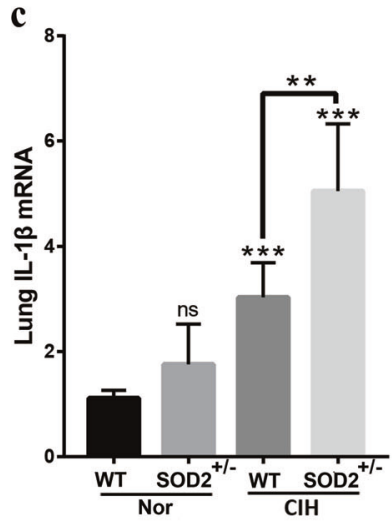

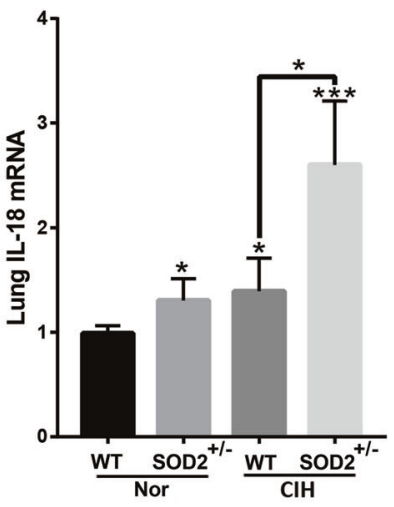

d
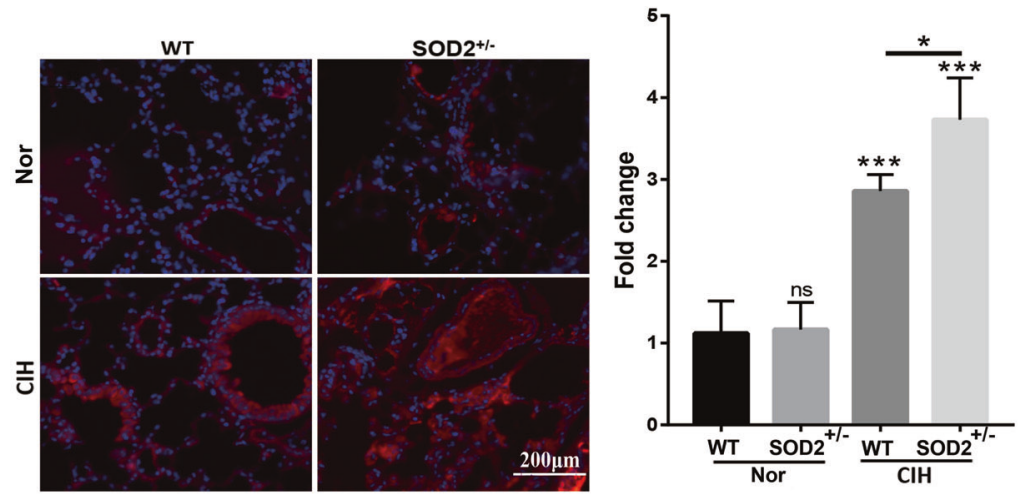

Fig. 3 Deficiency in SOD2 enhances $\mathrm{CIH}$-induced pulmonary inflammation and oxidative stress. a Morphological observations for the lungs from the WT, SOD2 ${ }^{+/-}, \mathrm{WT}+\mathrm{ClH}$, and $\mathrm{SOD}^{+/-}+\mathrm{ClH}$ groups. Representative light microscopy images of $\mathrm{HE}$ and Masson's trichrome staining are shown. b Pathological scoring results for lung sections from the four groups. c Quantitative reverse transcriptase polymerase chain reaction (RT-PCR) results for the interleukin (IL)- $1 \beta$ and IL-18 mRNA levels in lung lysates. $\mathbf{d}$ Immunohistochemical evaluation of ROS in mouse lungs from the four groups. The density of red fluorescence indicates the ROS level. Scale bar: $50 \mu \mathrm{m}$. Graph bars represent the mean \pm SEM from six mice per group. ${ }^{*} P<0.05,{ }^{*} P<0.01$, and ${ }^{* * *} P<0.001$ compared with the WT group. CIH chronic intermittent hypoxia, HE hematoxylin and eosin.

CD11c to analyze monocytes $\left(C D 11 b^{+}\right.$Ly6C $\left.^{+}\right)$, interstitial macrophages (IMs, F4/80 ${ }^{+} \mathrm{CD} 11 \mathrm{c}^{-}$), and alveolar macrophages (AMs,

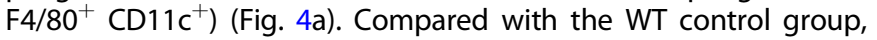
the $\mathrm{CIH}$ WT group had increased numbers of monocytes $(6.528 \% \pm$ $0.278 \%$ vs. $4.89 \% \pm 0.409 \%$, respectively, $P<0.05)$ and IMs $(13.82 \% \pm 0.39 \%$ vs. $10.22 \% \pm 0.78 \%$, respectively, $P<0.01)$, and the $\mathrm{CIH} \mathrm{SOD} 2^{+/-}$group seemed to have a further exacerbation of monocyte $(8.633 \% \pm 0.28 \%)$ and $I M(15.57 \% \pm 0.54 \%)$ infiltration into the lungs under the $\mathrm{ClH}$ conditions (Fig. 4b, c). In contrast, our flow cytometry analysis of AMs in the $\mathrm{ClH}$ lung tissue samples did not show a difference between the WT and SOD $2^{+/-}$groups with or without $\mathrm{ClH}$ exposure (Fig. 4d).

IH induces NLRP3 inflammasome activation in RAW264.7 cells Regarding the influence of $\mathrm{ClH}$ exposure on the immune response in vivo, we further investigated the molecular mechanism 

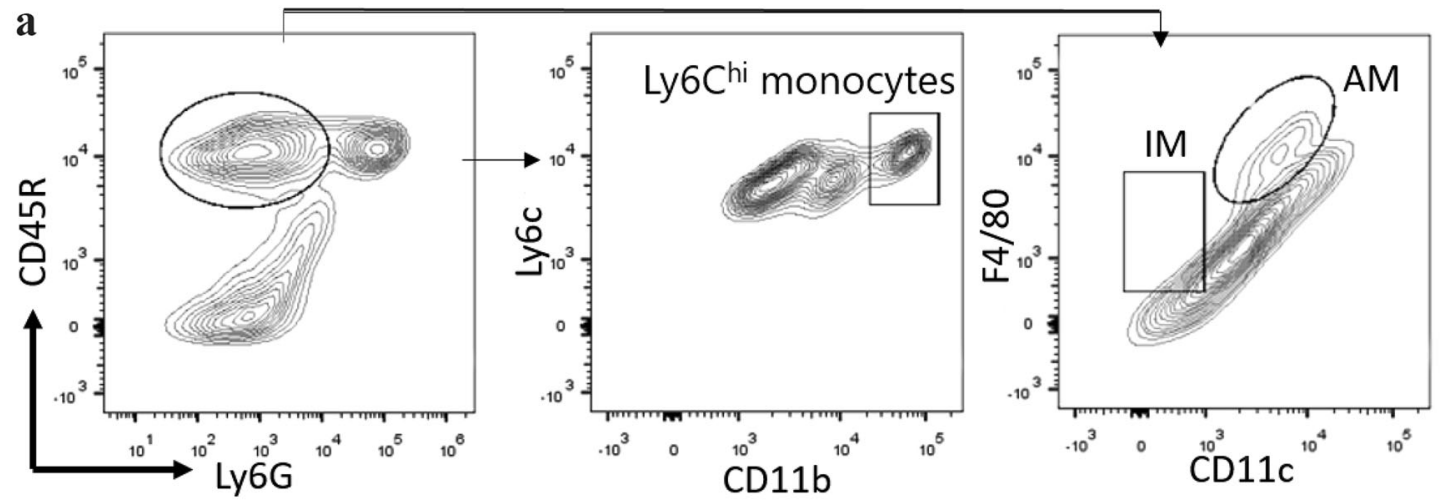

b

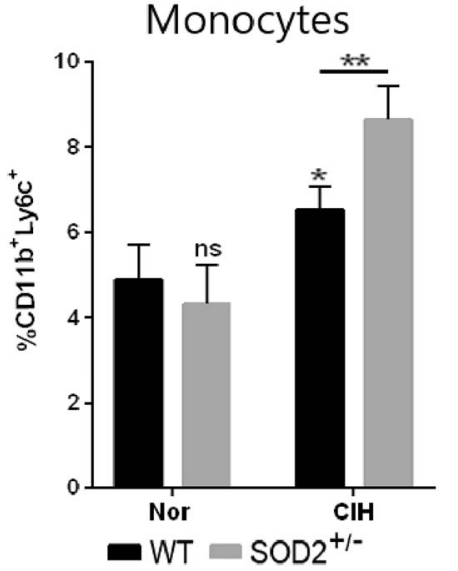

c

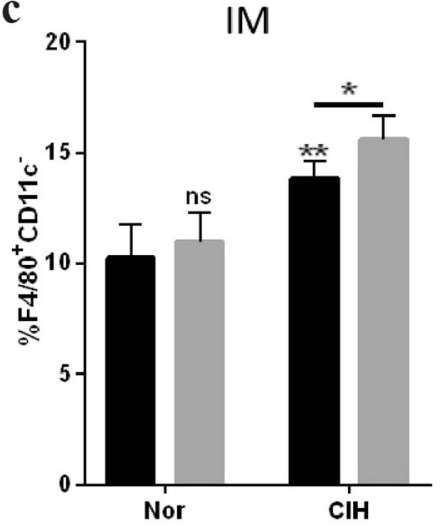

d

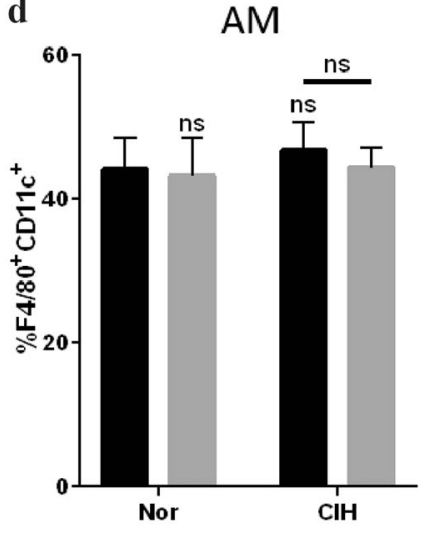

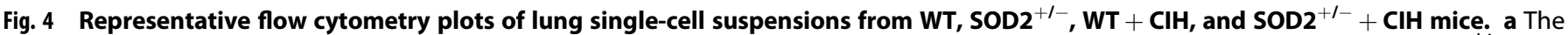
gating strategy for $\mathrm{CD}_{4} 5^{+}$myeloid lung cells to characterize pulmonary populations. The percentages of $\mathbf{b}$ monocytes (CD11 $\mathrm{b}^{+} \mathrm{Ly} 6 \mathrm{C}^{\mathrm{hi}}$ ), $\mathbf{c} \mathrm{F} 4 /$

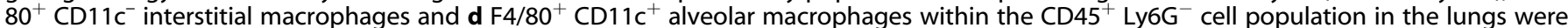
statistically analyzed. The mean \pm SEM is shown ( $n=6$ in each group). ${ }^{*} P<0.05$ and ${ }^{* *} P<0.01$ compared with the WT group. ClH chronic intermittent hypoxia, IMs interstitial macrophages, AMs alveolar macrophages.

underlying the $\mathrm{IH}$-mediated macrophage response in vitro. In the current study, IH exposure significantly increased the percentage of apoptotic cells, and as the time of $\mathrm{IH}$ exposure was extended, the percentage of apoptotic cells was increased (Fig. 5a, b). In addition, the content of mtROS was increased in RAW264.7 cells after exposure to the $\mathrm{IH}$ conditions (Fig. 5c, d). Western blot analysis showed that the expression of NLRP3, cleaved caspase-1 and ASC was significantly increased following $\mathrm{IH}$ exposure (Fig. 5e, f). These data suggested that $\mathrm{IH}$ increased mtROS production and NLRP3 inflammasome activation.

SOD2 knockdown triggers NLRP3 activation in RAW264.7 cells To further assess whether SOD2 modulates mtROS and NLRP3 activation in RAW264.7 cells under IH conditions, we transfected RAW264.7 cells with LV-shSOD2 and cultured them under Nor or IH conditions as described in the Methods section. The SOD2 knockdown efficiency was $74.5 \%$ (Fig. 6a, b). We observed that the inhibition of SOD2 by LV-shSOD2 induced apoptosis in the RAW264.7 cells even under the Nor conditions (vs. LV-NC control cells, $P<0.01$ ), and IH further enhanced apoptosis in LV-shSOD2transfected cells. In contrast, mito-TEMPO supplementation significantly reduced apoptosis in IH cells (vs. LV-NC IH cells, $P<0.001$, Fig. 6c, d). Then, we assessed whether SOD2 knockdown regulates mitochondrial membrane potential. In cells with low membrane potential, JC-1 showed green fluorescence instead of red fluorescence. Our results showed that in LV-shSOD2transfected cells with or without $\mathrm{IH}$ exposure, the density of green fluorescence was increased compared with that in control cells, indicating a decreased mitochondrial membrane potential.
In $\mathrm{IH}$ cells treated with mito-TEMPO, the density of green fluorescence was downregulated (vs. NC-LV IH cells, $P<0.01$, Fig. $6 \mathrm{e}-\mathrm{g}$ ).

Notably, the same tendency was observed for mtROS production (Fig. $7 a$ ). IL-1 $\beta$ mRNA and IL- $1 \beta$ protein levels in supernatants were obviously increased after $\mathrm{IH}$ exposure and decreased after treatment with mito-TEMPO (Fig. 7b, c). Likewise, Western blot analysis showed that the expression of NLRP3, cleavage caspase-1 and ASC was significantly increased following $\mathrm{IH}$ exposure and further elevated in LV-shSOD2-transfected cells. However, mitoTEMPO treatment markedly attenuated NLRP3 and ASC activation compared with $\mathrm{IH}$ treatment (Fig. 7d, e). Taken together, these data indicate that SOD2 deficiency can induce mitochondrial dysfunction and NLRP3 activation, while mito-TEMPO reverses IHinduced damage in macrophages, suggesting that mito-TEMPO supplement can reduce the strong production of reactive oxygen substances induced by $\mathrm{IH}$ within target cells.

\section{DISCUSSION}

In this study, we investigated the roles of SOD2 in $\mathrm{ClH}$-induced pulmonary inflammation and vascular remodeling. For the first time, we demonstrate that heterozygous SOD2 deletion counteracts $\mathrm{ClH}$-induced oxidative stress damage and manipulates the immune response by affecting macrophage subsets, thus contributing to lung injury. In detail, SOD2 ${ }^{+/-}$contributed to the $\mathrm{ClH}$-induced accumulation of infiltrating macrophage clusters accompanied by oxidative stress activation. Notably, we revealed that $\mathrm{IH}$-induced mtROS production and NLRP3 inflammasome 


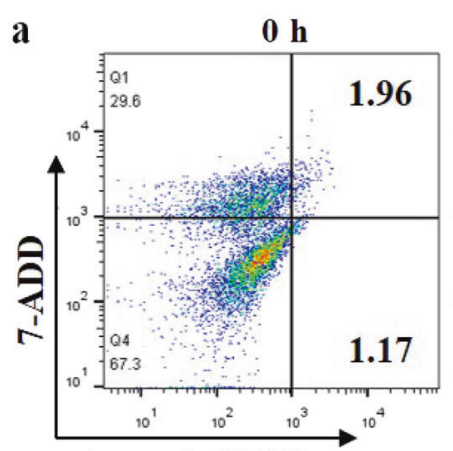

Annexin V-PE

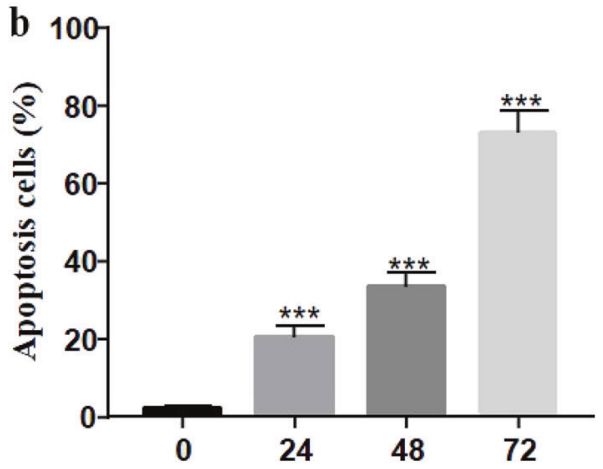

Duration of CIH treatment (h)
$24 \mathrm{~h}$

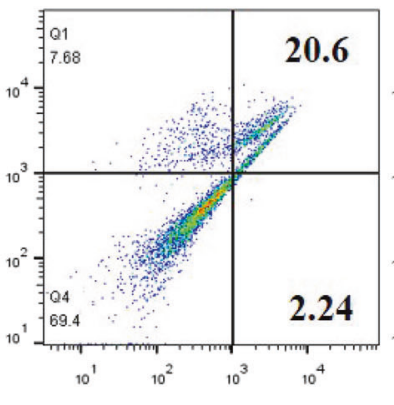

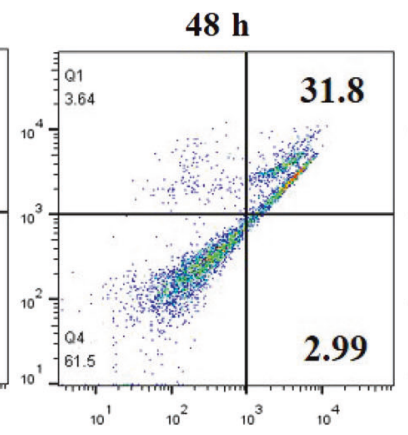

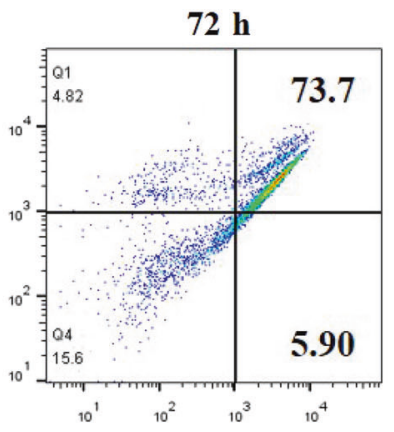

c

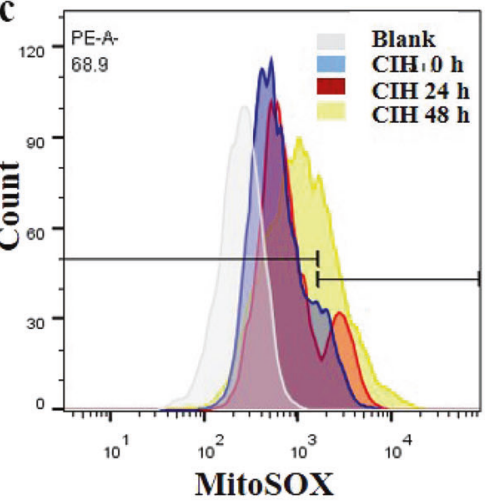

d

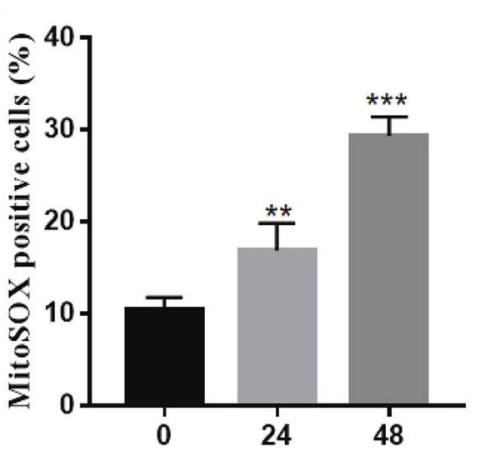

Duration of CIH treatment (h)

e
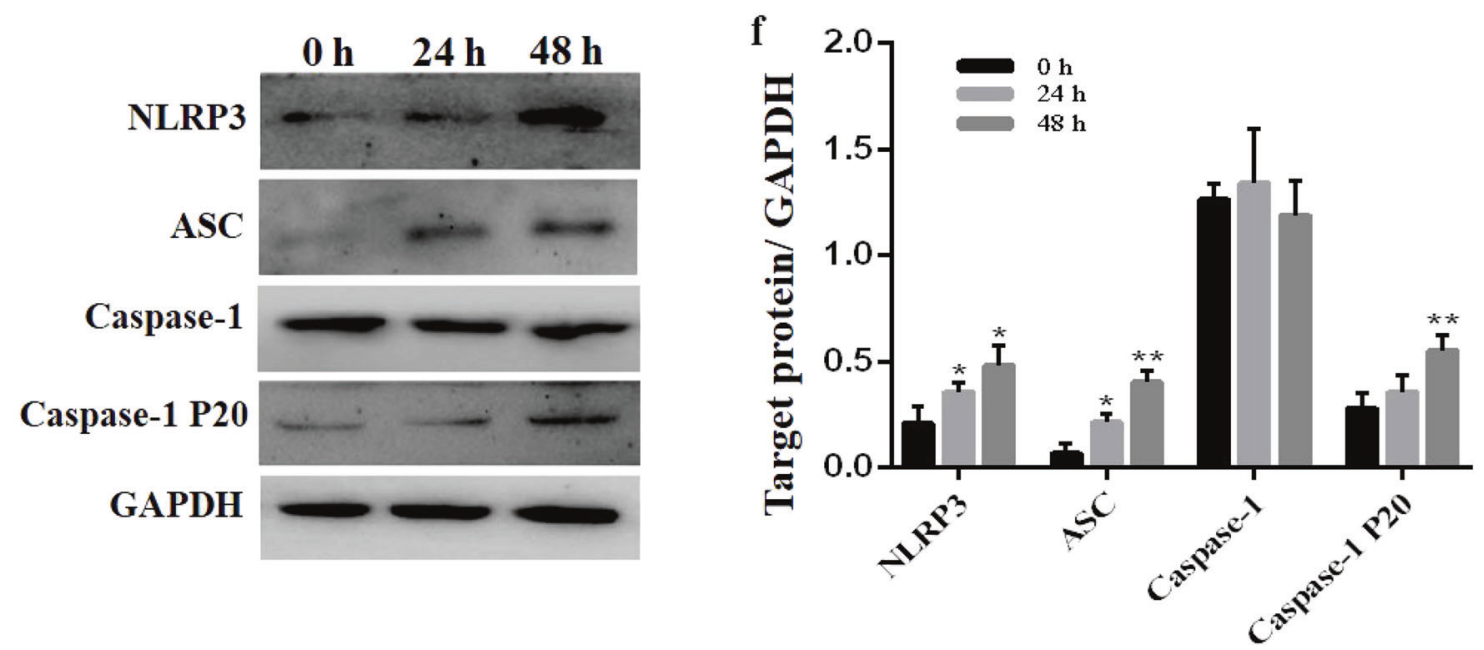

Fig. 5 IH induces apoptosis and mitochondrial oxidative stress in RAW264.7 cells. a Following IH exposure of RAW264.7 cells for 0, 24,48 or $72 \mathrm{~h}$, the cells were collected, stained using annexin V-PE/7-ADD and assessed by FACS analysis. b Quantitative analysis of apoptotic cell numbers is shown. c Flow cytometric analysis of cells treated with MitoSOX after IH exposure for 0, 24 or $48 \mathrm{~h}$ is shown. $\mathbf{d}$ Quantitative analysis of MitoSOX-positive cells is shown. e The protein expression of NLRP3 and ASC was detected by Western blot analysis. $\mathbf{f}$ Quantification of relative protein expression was performed by densitometric analysis, and GAPDH acted as an internal control. All data are presented as the mean \pm SEM. ${ }^{*} P<0.05,{ }^{* *} P<0.01$ and ${ }^{* *} P<0.001$ compared with the control group. Every experiment was repeated at least three times. IH intermittent hypoxia.

activation were further aggravated by SOD2 knockdown, but these effects of IH were markedly suppressed by mito-TEMPO in RAW264.7 cells. Our findings suggest that SOD2 deficiency contributes to the pathogenesis of $\mathrm{ClH}$-induced lung injury. Therefore, targeting SOD2 may potentially represent a therapeutic strategy for OSA patients that functions by limiting the $\mathrm{ClH}$ induced cascades of proinflammatory cytokines and related tissue injury.

In adults, OSA is highly and increasingly prevalent. OSA is associated with cardiovascular morbidities, insulin resistance, neural injury, and accelerated mortality [27]. In recent years, accumulating evidence has also suggested bidirectional relationships between OSA and lung diseases (COPD, asthma, interstitial lung diseases and $\mathrm{PAH}$ ), whereby each disorder deleteriously influences the other [28]. ROS generation, followed by immune dysfunction and chronic inflammation, constitutes one of the major mechanisms acting as an intermediary pathogenic link between OSA and target-organ damage [29]. Our previous study showed that $\mathrm{ClH}$ for 4 weeks increased ROS production and vascular remodeling in rat lungs. Notably, the overproduction of 
a

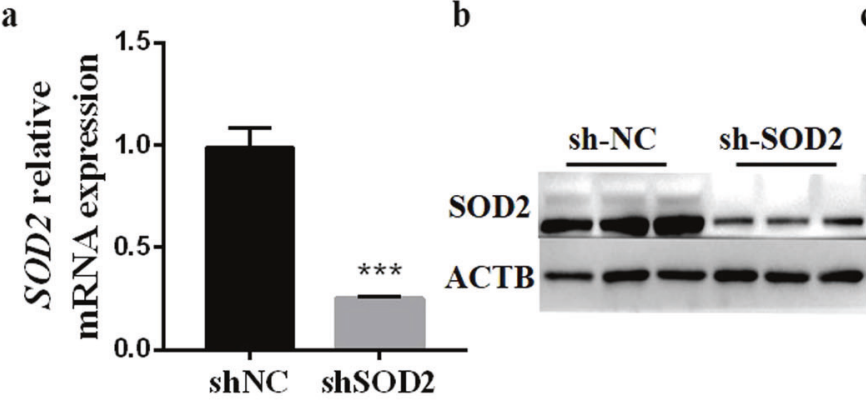

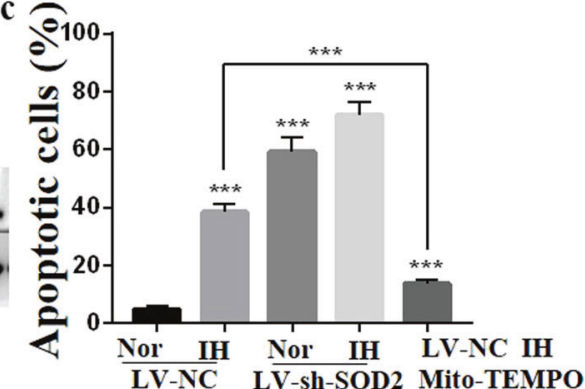

d
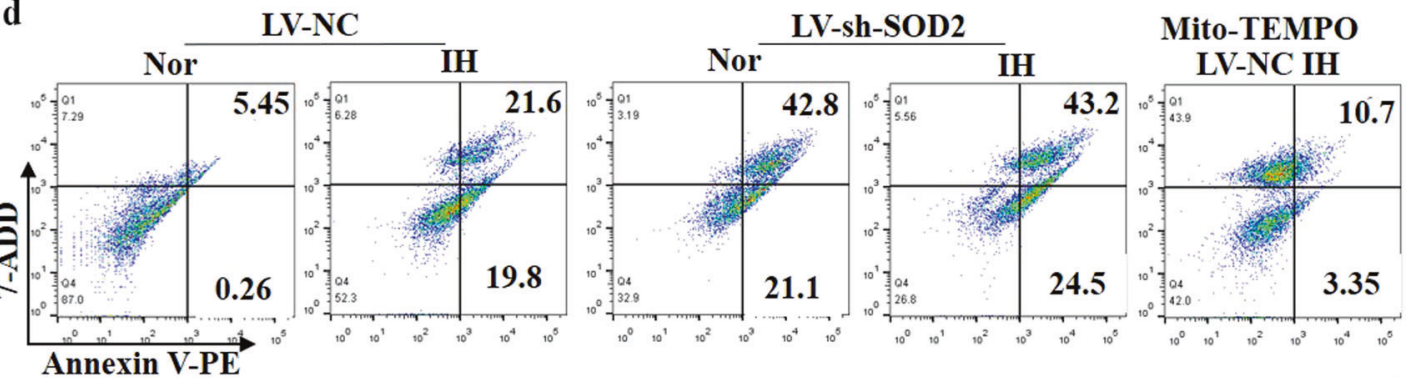

e
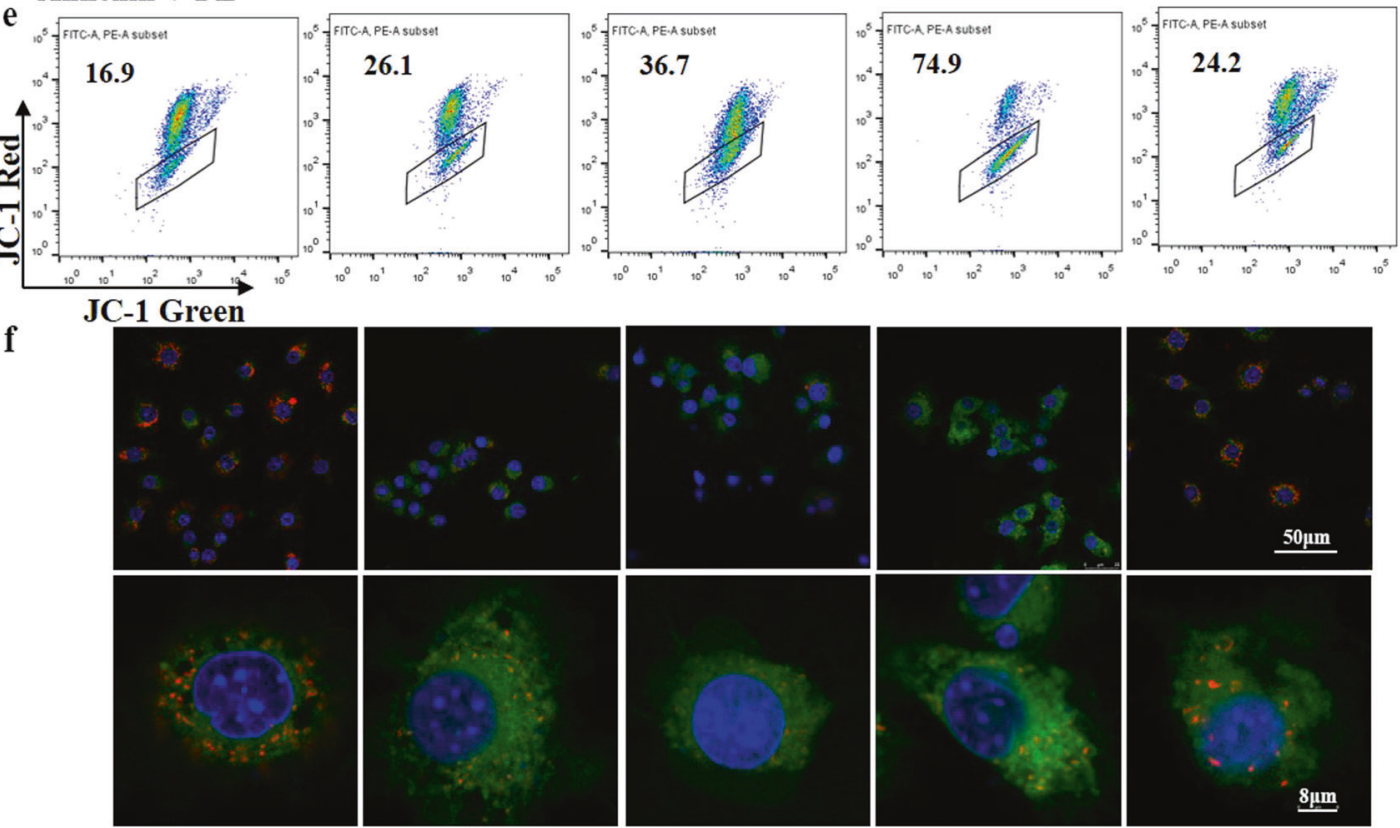

g
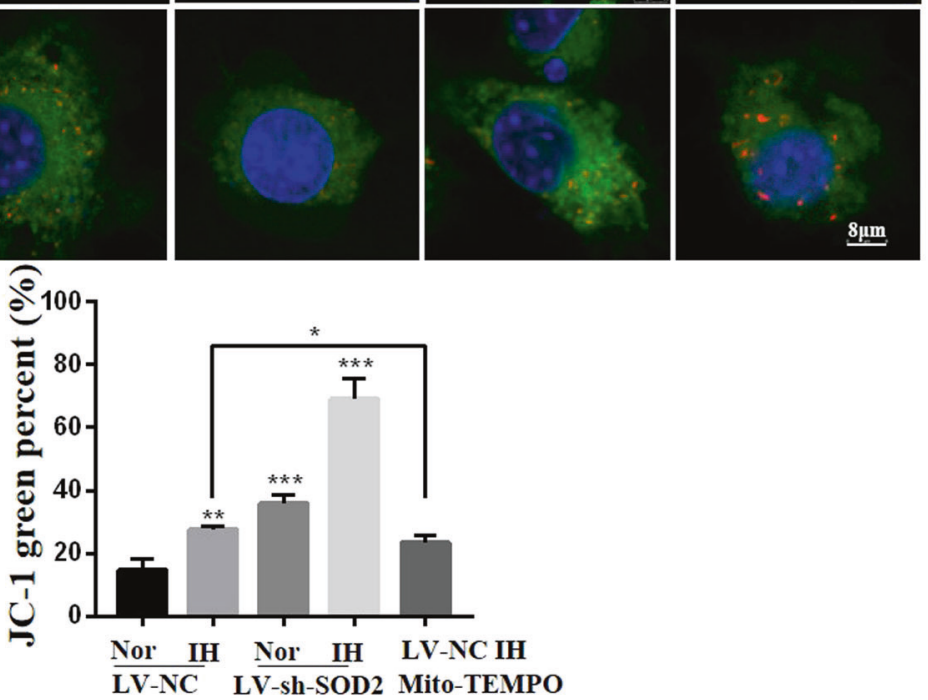

Fig. 6 Detection of apoptosis and mitochondrial membrane potential in LV-NC- or LV-shSOD2-transfected RAW264.7 cells incubated for $48 \mathrm{~h}$ under Nor or IH conditions with or without mito-TEMPO (100 nM). a Raw264.7 cells were transfected with LV-NC or LV-shSOD2. Cell lysates were subjected to real-time PCR (a) and Western blotting for SOD2 (b). ACTB ( $\beta$-actin) was used as the loading control. c The percentage of apoptotic cells in five groups was detected by FACS analysis. d Representative graphs of flow cytometric analysis of apoptosis are shown. e Representative graphs of flow cytometric analysis after incubation with JC-1 are shown. $\mathbf{f}$ Merged confocal microscopy images of RAW254.7 cells show the colocalization of JC-1 aggregates (red fluorescence represents JC- 1 aggregates in healthy mitochondria) and JC- 1 monomers (green fluorescence represents JC-1 monomers, indicating mitochondrial membrane potential dissipation). $\mathbf{g}$ Changes in mitochondrial membrane potential are represented as the green fluorescence ratio. All data are presented as the mean \pm SEM. ${ }^{*} P<0.05,{ }^{* *} P<$ 0.01 and ${ }^{* * *} P<0.001$ compared with the control group. Every experiment was repeated at least three times. IH intermittent hypoxia. 


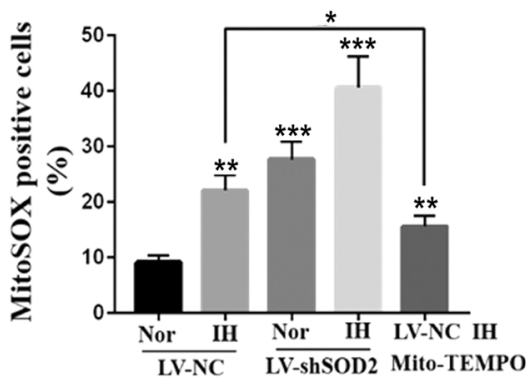

d

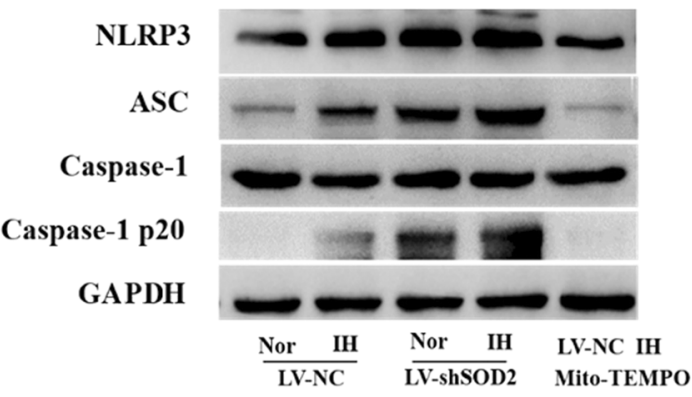

b

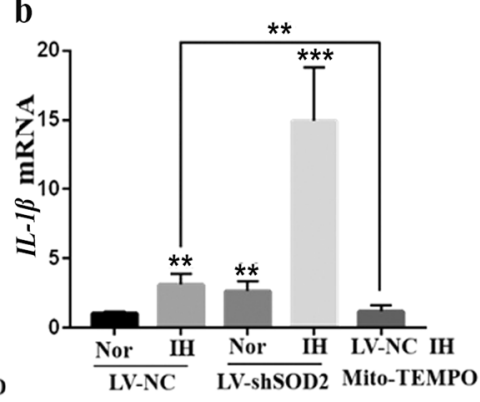

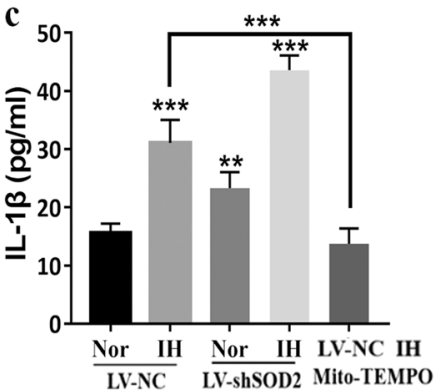

e

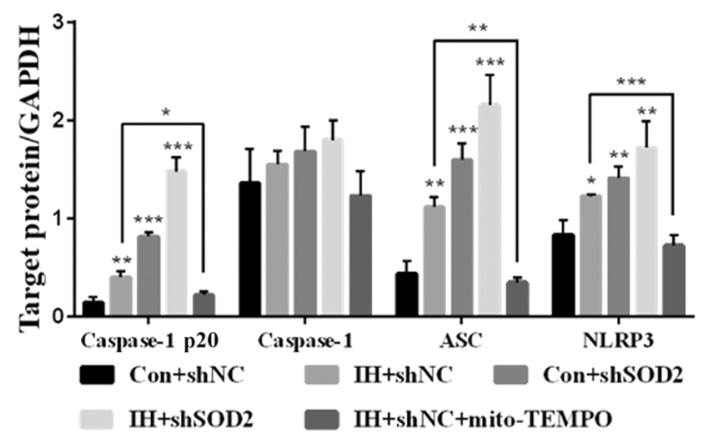

Fig. 7 Detection of mtROS and NLRP3 activation in LV-NC- or LV-shSOD2-transfected RAW264.7 cells incubated for $48 \mathrm{~h}$ under Nor or IH conditions with or without mito-TEMPO supplementation (100 $\mathrm{nM})$. a The percentage of mitoSOX-positive cells in the five groups was detected by FACS analysis. b RT-PCR results for IL-1 $\beta$ in cell lysates are shown. c Supernatants were analyzed for IL-1 $1 \beta$ by ELISA. d The protein expression of NLRP3 and ASC was detected by Western blot analysis. e Quantification of relative protein expression was performed by densitometric analysis, and GAPDH acted as an internal control. All data are presented as the mean \pm SEM. ${ }^{*} P<0.05$, ${ }^{* *} P<0.01$ and ${ }^{* * *} P<$ 0.001 compared with the control group. Every experiment was repeated at least three times. RT-PCR quantitative reverse transcriptasepolymerase chain reaction, IH intermittent hypoxia.

ROS in multiple cellular environments can specifically affect the sensitive modifying enzymes of the redox system and the repair proteins that play pivotal roles in the effects of $\mathrm{ClH}$.

SOD2 plays a critical role in protecting the mitochondria from oxidative stress [30]. Adult studies have reported markedly reduced SOD2 protein expression in PAH. Previous animal studies have demonstrated that SOD2 activity is inhibited in the lungs of PM2.5-exposed rat and lung fibrosis model animals [31, 32]. Finally, it has also been published that the mitochondria of SOD2 ${ }^{+/-}$mice show inhibition of respiration and early and rapid accumulation of mitochondrial oxidative damage [33]. Based on these studies, we hypothesized that these mice were relatively susceptible to oxidative stress injury in a $\mathrm{CIH}$ model of lung and pulmonary vascular disease. In the present work, OSA patients presented decreased SOD2 activity in the serum. SOD2 ${ }^{+/-}$mice had no detectable abnormal phenotype at baseline. However, after $\mathrm{CIH}$ exposure, the SOD2 protein level was further decreased in SOD2 ${ }^{+/-}$mice compared with WT mice. In addition, we noted that heterozygous SOD2 deletion caused histological damage and deteriorated arterial remodeling in $\mathrm{ClH}$ lungs, indicating the progression of inflammatory injury. A similar tendency was observed in other injury models. Wen and Garg reported that heterozygous SOD2 deletion exacerbated mtROS production in Chagas disease with peripheral and myocardial inflammatory infiltration [34]. Accordingly, the pathogenic mechanism can be attributed to increased mtROS expression and inflammatory cell infiltration.

Classically, activated macrophages in adipose, liver, and muscle tissues have been implicated in many conditions [35]. Despite numerous pulmonary comorbidities, the effects of macrophages on $\mathrm{ClH}$ lungs are less known, and the conclusions of available research are controversial. Romanova and Makarova reported that 1.5 months of $\mathrm{CIH}$ decreased the relative levels of alveolar macrophages and monocytes in the bronchoalveolar lavage fluid (BALF) of rats [36]. However, some studies have suggested that alveolar macrophages are active in OSA patients [37] and that $\mathrm{ClH}$ augments the frequency of $\mathrm{CD}^{+} 8^{+}$macrophages in mouse lungs; furthermore, IL-1 $\beta$ expression is significantly elevated in OSA patient BALF and $\mathrm{CIH}$ mouse lung tissues [38]. Our data indicated that increases in the levels of F4/ $80^{+} \mathrm{CD}_{11 \mathrm{c}^{-}}$interstitial macrophages and $\mathrm{CD}_{11 \mathrm{~b}^{+}} \mathrm{Ly}_{6 \mathrm{C}} \mathrm{hi}^{\mathrm{i}}$ monocytes were elicited by $\mathrm{CIH}$ and SOD2 deficiency exacerbated these tendencies.

The contributions of ROS signaling to the priming and activation of the NLRP3 inflammasome have been actively explored [39]. Conflicting findings have been reported, with some studies showing that ROS signaling is only necessary for NLRP3 inflammasome priming, whereas others show that oxidized mitochondrial DNA, as a consequence of excess ROS, can function as a damage-associated molecular pattern and directly bind to and activate the NLRP3 inflammasome [40]. Here, we showed that IH increased NLRP3 protein levels. The lack of SOD2 resulted in the amplification of NLRP3 inflammasome activation via mtROS signaling.

In conclusion, emerging evidence strongly supports the conclusion that SOD2 has an ameliorative effect on the activation of the NLRP3 inflammasome in both humans and mice $[10,39]$, and this effect appears to function in part by controlling mtROS levels. In this study, we provide the first evidence that SOD2 deficiency increases pulmonary inflammation and deteriorates vascular remodeling and demonstrate that mtROS/NLRP3 inflammasome activation is involved in $\mathrm{ClH}$ induced tissue damage. Hence, knowledge about oxidative stress in target cells and organs following $\mathrm{ClH}$ exposure may facilitate the development of strategies for complications associated with OSA. 


\section{ACKNOWLEDGEMENTS}

This work was supported by grants from The National Key Research and Development Program of China (No. 2018YFC1313600) and the National Natural Science Foundation of China (Nos. 81570081 and 81770083).

\section{AUTHOR CONTRIBUTIONS}

SYH conceived and designed the experiments; JQS, SYH, FCP and LZL performed the research; LX, WXD and XW analyzed the data; JQS and LYJ wrote the paper; and SQL contributed to manuscript preparation. SQL approved the final version to be submitted.

\section{ADDITIONAL INFORMATION}

Competing interests: The authors declare no competing interests.

\section{REFERENCES}

1. Sanchez-de-la-Torre M, Campos-Rodriguez F, Barbe F. Obstructive sleep apnoea and cardiovascular disease. Lancet Respir Med. 2013;1:61-72.

2. Drager LF, Togeiro SM, Polotsky VY, Lorenzi-Filho G. Obstructive sleep apnea: a cardiometabolic risk in obesity and the metabolic syndrome. J Am Coll Cardiol. 2013;62:569-76.

3. Torres G, Sanchez-de-la-Torre M, Barbe F. Relationship between OSA and hypertension. Chest. 2015;148:824-32.

4. Bucks RS, Olaithe M, Rosenzweig I, Morrell MJ. Reviewing the relationship between OSA and cognition: where do we go from here? Respirology. 2017;22:1253-61.

5. Patterson KC, Huang F, Oldham JM, Bhardwaj N, Hogarth DK, Mokhlesi B. Excessive daytime sleepiness and obstructive sleep apnea in patients with sarcoidosis. Chest. 2013;143:1562-8.

6. Lal C, Medarov BI, Judson MA. Interrelationship between sleep-disordered breathing and sarcoidosis. Chest. 2015;148:1105-14.

7. Wong HS, Williams AJ, Mok Y. The relationship between pulmonary hypertension and obstructive sleep apnea. Curr Opin Pulm Med. 2017;23:517-21.

8. Eisele HJ, Markart P, Schulz R. Obstructive sleep apnea, oxidative stress, and cardiovascular disease: evidence from human studies. Oxid Med Cell Longev. 2015;2015:608438.

9. Racanelli AC, Kikkers SA, Choi AMK, Cloonan SM. Autophagy and inflammation in chronic respiratory disease. Autophagy. 2018;14:221-32.

10. Yang $Y$, Wang $H$, Kouadir $M$, Song $H$, Shi F. Recent advances in the mechanisms of NLRP3 inflammasome activation and its inhibitors. Cell Death Dis. 2019;10:128.

11. Wang $\mathrm{H}$, Lv $\mathrm{C}$, Wang $\mathrm{S}$, Ying $\mathrm{H}$, Weng $\mathrm{Y}, \mathrm{Yu}$ W. NLRP3 inflammasome involves in the acute exacerbation of patients with chronic obstructive pulmonary disease. Inflammation. 2018:41:1321-33.

12. Sayan M, Mossman BT. The NLRP3 inflammasome in pathogenic particle and fibre-associated lung inflammation and diseases. Part Fibre Toxicol. 2016;13:51.

13. Wang L, Zha B, Shen Q, Zou H, Cheng C, Wu H, et al. Sevoflurane inhibits the Th2 response and NLRP3 expression in murine allergic airway inflammation. J Immunol Res. 2018;2018:9021037.

14. Wu X, Chang SC, Jin J, Gu W, Li S. NLRP3 inflammasome mediates chronic intermittent hypoxia-induced renal injury implication of the microRNA-155/ FOXO3a signaling pathway. J Cell Physiol. 2018;233:9404-15.

15. Bresciani G, da Cruz IB, Gonzalez-Gallego J. Manganese superoxide dismutase and oxidative stress modulation. Adv Clin Chem. 2015;68:87-130.

16. Seo YS, Kim HS, Lee AY, Chun JM, Kim SB, Moon BC, et al. Codonopsis lanceolata attenuates allergic lung inflammation by inhibiting Th2 cell activation and augmenting mitochondrial ROS dismutase (SOD2) expression. Sci Rep. 2019;9:2312.

17. Liu YD, Yu L, Ying L, Balic J, Gao H, Deng NT, et al. Toll-like receptor 2 regulates metabolic reprogramming in gastric cancer via superoxide dismutase 2 . Int J Cancer. 2018;144:3056-69.

18. Van Remmen H, Williams MD, Guo Z, Estlack L, Yang H, Carlson EJ, et al. Knockout mice heterozygous for Sod2 show alterations in cardiac mitochondrial function and apoptosis. Am J Physiol Heart Circ Physiol. 2001;281:H1422-32.

19. Epperly MW, Epstein CJ, Travis EL, Greenberger JS. Decreased pulmonary radiation resistance of manganese superoxide dismutase (MnSOD)-deficient mice is corrected by human manganese superoxide dismutase-Plasmid/Liposome (SOD2-PL) intratracheal gene therapy. Radiat Res. 2000;154:365-74.

20. Hemnes AR, Rathinasabapathy A, Austin EA, Brittain EL, Carrier EJ, Chen X, et al. A potential therapeutic role for angiotensin-converting enzyme 2 in human pulmonary arterial hypertension. Eur Respir J. 2018;51:1702638.

21. Traba J, Geiger SS, Kwarteng-Siaw M, Han K, Ra OH, Siegel RM, et al. Prolonged fasting suppresses mitochondrial NLRP3 inflammasome assembly and activation via SIRT3-mediated activation of superoxide dismutase 2. J Biol Chem. 2017;292:12153-64.

22. Panchanathan R, Liu H, Choubey D. Hypoxia primes human normal prostate epithelial cells and cancer cell lines for the NLRP3 and AIM2 inflammasome activation. Oncotarget. 2016;7:28183-94.

23. Folco EJ, Sukhova GK, Quillard T, Libby P. Moderate hypoxia potentiates interleukin-1beta production in activated human macrophages. Circ Res. 2014;115:875-83.

24. Hao S, Jiang L, Fu C, Wu X, Liu Z, Song J, et al. 2-Methoxyestradiol attenuates chronic-intermittent-hypoxia-induced pulmonary hypertension through regulating microRNA-223. J Cell Physiol. 2019;234:6324-35.

25. Fu C, Hao S, Xu X, Zhou J, Liu Z, Lu H, et al. Activation of SIRT1 ameliorates LPSinduced lung injury in mice via decreasing endothelial tight junction permeability. Acta Pharmacol Sin. 2019;40:630-41.

26. Pugliese SC, Kumar S, Janssen WJ, Graham BB, Frid MG, Riddle SR, et al. A timeand compartment-specific activation of lung macrophages in hypoxic pulmonary hypertension. J Immunol. 2017;198:4802-12.

27. Peppard PE, Young T, Barnet JH, Palta M, Hagen EW, Hla KM. Increased prevalence of sleep-disordered breathing in adults. Am J Epidemiol. 2013;177:1006-14.

28. Teodorescu M, Barnet JH, Hagen EW, Palta M, Young TB, Peppard PE. Association between asthma and risk of developing obstructive sleep apnea. JAMA 2015;313:156-64.

29. Dewan NA, Nieto FJ, Somers VK. Intermittent hypoxemia and OSA: implications for comorbidities. Chest. 2015;147:266-74.

30. Lebovitz RM, Zhang H, Vogel H, Cartwright J Jr, Dionne L, Lu N, et al. Neurodegeneration, myocardial injury, and perinatal death in mitochondrial superoxide dismutase-deficient mice. Proc Natl Acad Sci USA. 1996;93:9782-7.

31. Li R, Kou X, Geng H, Xie J, Yang Z, Zhang Y, et al. Effect of ambient PM(2.5) on lung mitochondrial damage and fusion/fission gene expression in rats. Chem Res Toxicol. 2015;28:408-18.

32. Jablonski RP, Kim SJ, Cheresh P, Williams DB, Morales-Nebreda L, Cheng Y, et al. SIRT3 deficiency promotes lung fibrosis by augmenting alveolar epithelial cell mitochondrial DNA damage and apoptosis. Faseb J. 2017;31:2520-32.

33. Kokoszka JE, Coskun $\mathrm{P}$, Esposito LA, Wallace DC. Increased mitochondrial oxidative stress in the SOD2 (+/-) mouse results in the age-related decline of mitochondrial function culminating in increased apoptosis. Proc Natl Acad Sci USA. 2001;98:2278-83.

34. Wen JJ, Garg NJ. Manganese superoxide dismutase deficiency exacerbates the mitochondrial ROS production and oxidative damage in Chagas disease. PLoS Negl Trop Dis. 2018;12:e0006687.

35. Murphy AM, Thomas A, Crinion SJ, Kent BD, Tambuwala MM, Fabre A, et al. Intermittent hypoxia in obstructive sleep apnoea mediates insulin resistance through adipose tissue inflammation. Eur Respir J. 2017;49:1601731.

36. Romanova LK, Makarova LF. Bronchoalveolar lavage cells and mitotic activity of monocytes and macrophages in rats after long-term intermittent hypoxia. Bull Exp Biol Med. 2000;130:945-7.

37. Sharma S, Malur A, Marshall I, Huizar I, Barna BP, Pories W, et al. Alveolar mac rophage activation in obese patients with obstructive sleep apnea. Surgery. 2012;151:107-12.

38. Tuleta I, Stockigt F, Juergens UR, Pizarro C, Schrickel JW, Kristiansen G, et al Intermittent hypoxia contributes to the lung damage by increased oxidative stress, inflammation, and disbalance in protease/antiprotease system. Lung. 2016;194:1015-20.

39. Tschopp J, Schroder K. NLRP3 inflammasome activation: the convergence of multiple signalling pathways on ROS production? Nat Rev Immunol. 2010;10:210-5.

40. Shimada K, Crother TR, Karlin J, Dagvadorj J, Chiba N, Chen S, et al. Oxidized mitochondrial DNA activates the NLRP3 inflammasome during apoptosis. Immunity. 2012;36:401-14. 